\title{
Ulam's Type Stability of Involutional-Exponential Functional Equations
}

\author{
Jaeyoung Chung \\ Department of Mathematics, Kunsan National University, Gunsan 573-701, Republic of Korea \\ Correspondence should be addressed to Jaeyoung Chung; jychung@kunsan.ac.kr
}

Received 26 May 2014; Accepted 29 July 2014; Published 14 October 2014

Academic Editor: Ming Mei

Copyright (C) 2014 Jaeyoung Chung. This is an open access article distributed under the Creative Commons Attribution License, which permits unrestricted use, distribution, and reproduction in any medium, provided the original work is properly cited.

Let $S$ be a commutative semigroup, $f, g: S \rightarrow \mathbb{C}$ and $\sigma: S \rightarrow S$ an involution. In this paper we consider the stability of involutionexponential functional equations $|f(x+\sigma y)-g(x) f(y)| \leq \phi(x)[$ resp., $\phi(y)],|f(x+\sigma y)-f(x) g(y)| \leq \phi(x)[$ resp., $\phi(y)]$ for all $x, y \in S$, where $\phi: S \rightarrow \mathbb{R}^{+}$satisfies the growth condition: there exists $C>1$ such that $\lim _{k \rightarrow \infty} C^{-k} \phi(k x)=0$ for each $x \in S$. We also consider the stability of $L^{\infty}$-version $|f(x+\sigma y)-f(x) f(y)|_{L^{\infty}\left(\mathbb{R}^{2 n}\right)} \leq \epsilon$, where $f: \mathbb{R}^{n} \rightarrow \mathbb{C}$ is a locally integrable function.

\section{Introduction}

Throughout this paper we denote by $S, \mathbb{R}, \mathbb{R}^{+}, \mathbb{C}, \mathbb{R}^{n}$, a commutative semigroup with an identity element, the set of real numbers, nonnegative real numbers, complex numbers, and the $n$-dimensional Euclidean space, respectively, and $\mathbb{R}_{0}^{n}=$ $\mathbb{R}^{n} \backslash\{0\}, \mathbb{C}_{0}=\mathbb{C} \backslash\{0\}, \phi: S \rightarrow \mathbb{R}^{+}, \epsilon \geq 0$. A function $m: S \rightarrow$ $\mathbb{C}$ is called exponential provided that $m(x+y)=m(x) m(y)$ for all $x, y \in S$, and $\sigma: S \rightarrow S$ is called an involution provided that $\sigma(x+y)=\sigma(x)+\sigma(y)$ and $\sigma(\sigma(x))=x$ for all $x, y \in S$. An exponential function $m: S \rightarrow \mathbb{C}$ is called $\sigma$-exponential if $m$ satisfies $m(\sigma x)=m(x)$ for all $x \in S$ and denote by $m_{\sigma}$ a $\sigma$-exponential function.

In [1], the following functional inequalities with involution are investigated:

$$
|f(x+\sigma y)-g(x) f(y)| \leq \phi(x)[\operatorname{resp} . \phi(y)], \quad \forall x, y \in S,
$$

$|f(x+\sigma y)-f(x) g(y)| \leq \phi(x)[$ resp. $\phi(y)], \quad \forall x, y \in S$.

As a result, all unbounded functions $f, g$ satisfying the inequalities (1) and (2) are exactly described only when $\phi$ is a constant function while only one of unbounded functions $f, g$ satisfying each of (1) and (2) is exactly described when $\phi$ is an arbitrary unbounded function.
In this paper we investigate the functional inequalities (1) and (2) by imposing some growth conditions on $\phi, f$, or $g$. First, we introduce the condition on $h: S \rightarrow \mathbb{C}$ :

$$
\inf _{x \in S} \frac{1+\phi(x)}{|h(x)|}=0,
$$

where $h$ will stand for $f$ and $g$.

Secondly, we introduce the condition on $\phi$; there exists $C>1$ such that

$$
\lim _{k \rightarrow \infty} C^{-k} \phi(k x)=0
$$

for all $x \in S$.

As a result, we completely determine $f$ and $g$ satisfying each of the inequalities (1) and (2): if $g$ satisfies (3) [resp., $f$ satisfies (3)] or $\phi$ satisfies (4), then $(f, g)$ satisfying (1) [resp., (2)] are of the form

$$
f(x)=f(0) m_{\sigma}(x),
$$

$$
\begin{aligned}
g(x)= & m_{\sigma}(x) \\
& \times[\text { resp. } f(x)=f(0) m(x), g(x)=m(\sigma x)]
\end{aligned}
$$

for all $x \in S$, where $m_{\sigma}$ is a $\sigma$-exponential function and $m$ is an exponential function. 
As an application of our result, we determine all unbounded functions $f, g: \mathbb{R}_{0}^{2} \rightarrow \mathbb{R}$ satisfying the functional inequalities

$$
\begin{aligned}
& |f(u x+v y, u y-v x)-g(x, y) f(u, v)| \\
& \quad \leq \phi(x, y)[\phi(u, v)], \\
& |f(u x+v y, u y-v x)-f(x, y) g(u, v)| \\
& \quad \leq \phi(x, y)[\phi(u, v)]
\end{aligned}
$$

for all $(x, y),(u, v) \in \mathbb{R}_{0}^{2}$, where $f, g$ satisfy (3) or $\phi: \mathbb{R}_{0}^{2}$ $\rightarrow \mathbb{R}^{+}$satisfies (4) (see [2-5] for related equations) and determine all unbounded functions $f, g: \mathbb{R}_{0}^{4} \rightarrow \mathbb{R}$ satisfying the functional inequalities

$$
\begin{gathered}
\mid f\left(x_{1}, y_{1}, u_{1}, v_{1}\right) g\left(x_{2}, y_{2}, u_{2}, v_{2}\right) \\
-f\left(x_{1} x_{2}+y_{1} y_{2}+u_{1} u_{2}+v_{1} v_{2}, x_{1} y_{2}-y_{1} x_{2}\right. \\
+u_{1} v_{2}-v_{1} u_{2}, x_{1} u_{2}-y_{1} v_{2}-u_{1} x_{2} \\
\left.+v_{1} y_{2}, x_{1} v_{2}+y_{1} u_{2}-u_{1} y_{2}-v_{1} x_{2}\right) \mid \\
\leq \psi\left(x_{1}, y_{1}, u_{1}, v_{1}\right)\left[\psi\left(x_{2}, y_{2}, u_{2}, v_{2}\right)\right]
\end{gathered}
$$

for all $x_{1}, y_{1}, u_{1}, v_{1}, x_{2}, y_{2}, u_{2}$, and $v_{2} \in \mathbb{R}$, where $f, g$ satisfy (3) or $\psi: \mathbb{R}_{0}^{4} \rightarrow \mathbb{R}^{+}$satisfies (4) (see $[2,4]$ for related equations). Finally, we consider the stability of $L^{\infty}$-version

$$
|f(x+\sigma y)-f(x) f(y)|_{L^{\infty}\left(\mathbb{R}^{2 n}\right)} \leq \epsilon,
$$

where $f: \mathbb{R}^{n} \rightarrow \mathbb{C}$ is a locally integrable function. As a result, we prove that every unbounded solution $f$ (i.e., $\|f\|_{L^{\infty}\left(\mathbb{R}^{n}\right)}=$ $\infty)$ of (9) satisfies

$$
f(x)=m\left(\frac{x+\sigma x}{2}\right)
$$

for almost every $x \in \mathbb{R}^{n}$, where $m: \mathbb{R}^{n} \rightarrow \mathbb{C}$ is an unbounded exponential function. Every bounded solution $f$ (i.e., $\left.\|f\|_{L^{\infty}\left(\mathbb{R}^{n}\right)}<\infty\right)$ satisfies

$$
\|f\|_{L^{\infty}\left(\mathbb{R}^{n}\right)} \leq \frac{1}{2}(1+\sqrt{1+4 \epsilon}) ;
$$

If $\epsilon<1 / 4$, then $f$ satisfies either

$$
\frac{1}{2}(1+\sqrt{1-4 \epsilon}) \leq\|f\|_{L^{\infty}\left(\mathbb{R}^{n}\right)} \leq \frac{1}{2}(1+\sqrt{1+4 \epsilon})
$$

or

$$
\|f\|_{L^{\infty}\left(\mathbb{R}^{n}\right)} \leq \frac{1}{2}(1-\sqrt{1-4 \epsilon}) .
$$

We refer the reader to $[1,6-16]$ for related functional equations and their stabilities. We also refer the reader to [1719] for some recent developments on the issues of stability and superstability for functional equations.

\section{Stability of (1) and (2)}

In this section we investigate unbounded functions $f, g$ satisfying (1) and (2) when some of $f$ and $g$ satisfy (3) or $\phi$ satisfies (4). For bounded solutions of (1) and (2) we refer the reader to [1].

Lemma 1. Assume that $m: S \rightarrow \mathbb{C}$ is an unbounded exponential function and $\phi: S \rightarrow \mathbb{R}^{+}$satisfies (4). Then $m$ satisfies (3).

Proof. Since $m$ is unbounded, we can choose a $x_{0} \in S$ such that $\left|m\left(x_{0}\right)\right| \geq C$, where $C>1$ is the constant in (4). Since $\phi$ satisfies (4) we have

$$
\begin{aligned}
\lim _{k \rightarrow \infty} \frac{1+\phi\left(k x_{0}\right)}{\left|m\left(k x_{0}\right)\right|} & =\lim _{k \rightarrow \infty} \frac{1+\phi\left(k x_{0}\right)}{\left|m\left(x_{0}\right)\right|^{k}} \\
& \leq \lim _{k \rightarrow \infty}\left(C^{-k}+C^{-k} \phi\left(k x_{0}\right)\right)=0 .
\end{aligned}
$$

This completes the proof.

Theorem 2. Let $f, g: S \rightarrow \mathbb{C}$ be unbounded functions satisfying

$$
|f(x+\sigma y)-g(x) f(y)| \leq \phi(x)
$$

for all $x, y \in S$. Then $g$ is a $\sigma$-exponential function. In particular if $g$ satisfies (3) or $\phi$ satisfies (4), then there exists a $\sigma$ exponential function $m_{\sigma}: S \rightarrow \mathbb{C}$ such that

$$
f(x)=f(0) m_{\sigma}(x), \quad g(x)=m_{\sigma}(x)
$$

for all $x \in S$.

Proof. Choosing a sequence $y_{n} \in S, n=1,2,3, \ldots$, such that $\left|f\left(y_{n}\right)\right| \rightarrow \infty$ as $n \rightarrow \infty$, putting $y=y_{n}, n=1,2,3, \ldots$, in (15), dividing the result by $\left|f\left(y_{n}\right)\right|$, and letting $n \rightarrow \infty$ we have

$$
g(x)=\lim _{n \rightarrow \infty} \frac{f\left(x+\sigma y_{n}\right)}{f\left(y_{n}\right)}
$$

for all $x \in S$. Putting $x=0$ in (15) we have

$$
|f(\sigma y)-g(0) f(y)| \leq \phi(0)
$$

for all $y \in S$. Multiplying both sides of (17) by $g(y)$ and using (15), (17), and (18) we have

$$
\begin{aligned}
g(y) g(x) & =\lim _{n \rightarrow \infty} \frac{g(y) f\left(x+\sigma y_{n}\right)}{f\left(y_{n}\right)}=\lim _{n \rightarrow \infty} \frac{f\left(y+\sigma x+y_{n}\right)}{f\left(y_{n}\right)} \\
& =\lim _{n \rightarrow \infty} \frac{g(0) f\left(x+\sigma y+\sigma y_{n}\right)}{f\left(y_{n}\right)}=g(0) g(x+\sigma y)
\end{aligned}
$$

for all $x, y \in S$. Dividing (19) by $g(0)^{2}$ we have

$$
g_{0}(x) g_{0}(y)=g_{0}(x+\sigma y)
$$

for all $x, y \in S$, where $g_{0}(x)=g(x) / g(0)$. From (20) we have

$$
g(x)=g(0) m_{\sigma}(x)
$$


for some $\sigma$-exponential $m_{\sigma}$. If $g$ satisfies (3) or $\phi$ satisfies (4), then, by Lemma 1 , we can choose a sequence $x_{n} \in S, n=$ $1,2,3, \ldots$, such that $\left(1+\phi\left(x_{n}\right)\right) /\left|g\left(x_{n}\right)\right| \rightarrow 0$ as $n \rightarrow \infty$. Putting $x=x_{n}, n=1,2,3, \ldots$, in (15), dividing the result by $\left|g\left(x_{n}\right)\right|$, and letting $n \rightarrow \infty$ we have

$$
f(y)=\lim _{n \rightarrow \infty} \frac{f\left(x_{n}+\sigma y\right)}{g\left(x_{n}\right)}
$$

for all $y \in S$. Multiplying both sides of (22) by $g(x)$ and using (15), (18), and (22) we have

$$
\begin{aligned}
g(x) f(y) & =\lim _{n \rightarrow \infty} \frac{g(x) f\left(x_{n}+\sigma y\right)}{g\left(x_{n}\right)} \\
& =\lim _{n \rightarrow \infty} \frac{f\left(x+\sigma x_{n}+y\right)}{g\left(x_{n}\right)} \\
& =\lim _{n \rightarrow \infty} \frac{g(0) f\left(\sigma x+\sigma y+x_{n}\right)}{g\left(x_{n}\right)}=g(0) f(x+y)
\end{aligned}
$$

for all $x, y \in S$. Putting $y=0$ in (23) and dividing the result by $g(0)$ we have

$$
f(x)=f(0) g_{0}(x)=f(0) m_{\sigma}(x)
$$

for all $x \in S$. Putting $x=0$ in (15) and using (24) we have

$$
|f(0)(1-g(0))|\left|m_{\sigma}(y)\right| \leq \phi(0)
$$

for all $y \in S$. Since $m_{\sigma}$ is unbounded, from (25) we have $g(0)=1$. Now, from (21) and (24) we get (16). This completes the proof.

We denote by $c \cdot x$ the inner product of $c=\left(c_{1}, c_{2}, \ldots, c_{n}\right) \in$ $\mathbb{C}^{n}$ and $x=\left(x_{1}, x_{2}, \ldots, x_{n}\right) \in \mathbb{R}^{n}$ which is defined as $c \cdot x=$ $\sum_{j=1}^{n} c_{j} x_{j}$, and $\mathfrak{R} c=\left(\mathfrak{R} c_{1}, \ldots, \mathfrak{R} c_{n}\right)$, where $\mathfrak{R} c_{j}$ are the real parts of $c_{j}, j=1,2, \ldots, n$. It is easy to see that if $S$ is uniquely 2-divisible (i.e., for each $x \in S$ there exists a unique $y \in S$ such that $2 y=x$ ), then $m_{\sigma}$ is $\sigma$-exponential if and only if

$$
m_{\sigma}(x)=m\left(\frac{x+\sigma x}{2}\right), \quad x \in S
$$

for some exponential function $m: S \rightarrow \mathbb{C}$.

Corollary 3. Let $P(x), x \in \mathbb{R}^{n}$, be a polynomial. Suppose that $f, g: \mathbb{R}^{n} \rightarrow \mathbb{C}$ are unbounded function satisfying

$$
|f(x+\sigma y)-g(x) f(y)| \leq|P(x)|
$$

for all $x, y \in \mathbb{R}^{n}$. Then there exists a $\sigma$-exponential function $m_{\sigma}: \mathbb{R}^{n} \rightarrow \mathbb{C}$ such that

$$
f(x)=f(0) m_{\sigma}(x), \quad g(x)=m_{\sigma}(x)
$$

for all $x \in \mathbb{R}^{n}$. In particular if $g$ is continuous, then there exists $c \in \mathbb{C}^{n}, \mathfrak{R}(c+c \sigma) \neq 0$ such that

$$
f(x)=f(0) e^{(1 / 2)(c+c \sigma) \cdot x}, \quad g(x)=e^{(1 / 2)(c+c \sigma) \cdot x}
$$

for all $x \in \mathbb{R}^{n}$.
Proof. It is easy to see that $|P(x)|$ satisfies (4). Thus, by Theorem 2 we get (28). Assume that $g$ is continuous. It is well known that every continuous exponential functional $m$ : $\mathbb{R}^{n} \rightarrow \mathbb{C}$ is given by $m(x)=e^{c \cdot x}$ for some $c \in \mathbb{C}^{n}$. Thus, from (26) we have $m_{\sigma}(x)=e^{(1 / 2)(c+c \sigma) \cdot x}$ for all $x \in \mathbb{R}^{n}$, where $c \sigma$ denotes matrix multiplication. Thus, we get (29). This completes the proof.

Remark 4. Let $a, b \in \mathbb{R}^{n}$ be two nonzero vectors that are not parallel; that is, $b \neq r a$ for all $r \in \mathbb{R}$. Then, the hyperplane $b \cdot x=0$ is not parallel to $(b-a) \cdot x=0$ and hence there exists $x_{0} \in \mathbb{R}^{n}$ such that $b \cdot x_{0}>0$ and $(b-a) \cdot x_{0}>0$. If $b=t a$ for some $t \in \mathbb{R}$, then there exists $x_{0} \in \mathbb{R}^{n}$ such that $b \cdot x_{0}>0$ and $(b-a) \cdot x_{0}>0$ if and only if $t>1$. Thus, if $b \neq t a$ for all $t \leq 1$, then there exists $x_{0} \in \mathbb{R}^{n}$ such that $b \cdot x_{0}>0$ and $(b-a) \cdot x_{0}>0$.

Corollary 5. Let $\gamma \in \mathbb{R}^{n}$ be fixed. Suppose that $f, g: \mathbb{R}^{n} \rightarrow \mathbb{C}$ are unbounded continuous function satisfying

$$
|f(x+\sigma y)-g(x) f(y)| \leq e^{\gamma \cdot x}
$$

for all $x, y \in \mathbb{R}^{n}$. Then there exists $c \in \mathbb{C}^{n}, \mathfrak{R}(c+c \sigma) \neq 0$ such that $g(x)=e^{(1 / 2)(c+c \sigma) \cdot x}$ for all $x \in \mathbb{R}^{n}$. If $(1 / 2) \mathfrak{R}(c+c \sigma) \neq t \gamma$ for all $t \leq 1$, then one has

$$
f(x)=f(0) e^{(1 / 2)(c+c \sigma) \cdot x}, \quad g(x)=e^{(1 / 2)(c+c \sigma) \cdot x}
$$

for all $x \in \mathbb{R}^{n}$.

Proof. Recall that every continuous $\sigma$-exponential functional $m_{\sigma}: \mathbb{R}^{n} \rightarrow \mathbb{C}$ is given by

$$
m_{\sigma}(x)=e^{(1 / 2)(c+c \sigma) \cdot x}
$$

for all $x \in \mathbb{R}^{n}$, where $c \sigma$ denotes matrix multiplication. If $(1 / 2) \mathfrak{R}(c+c \sigma) \neq t \gamma$ for all $t \leq 1$, then by Remark 4 there exists $x_{0} \in \mathbb{R}^{n}$ such that

$$
\frac{1}{2}(c+c \sigma) \cdot x_{0}>0, \quad \frac{1}{2}(c+c \sigma) \cdot x_{0}>\gamma \cdot x_{0} .
$$

From (32) and (33) we have

$$
\lim _{k \rightarrow \infty} \frac{1+e^{\gamma \cdot k x_{0}}}{g\left(k x_{0}\right)}=\frac{1+e^{\gamma \cdot k x_{0}}}{e^{(1 / 2)(c+c \sigma) \cdot k x_{0}}}=0,
$$

which implies that $g$ satisfies the condition (3). Thus, we get (31). This completes the proof.

Theorem 6. Let $f, g: S \rightarrow \mathbb{C}$ be unbounded functions satisfying

$$
|f(x+\sigma y)-g(x) f(y)| \leq \phi(y)
$$

for all $x, y \in S$. Then there exists an unbounded $\sigma$-exponential function $m_{\sigma}: S \rightarrow \mathbb{C}$ such that $f(x)=f(0) m_{\sigma}(x)$ for all $x \in$ $S$. In particular if $f$ satisfies (3) or $\phi$ satisfies (4), then one has

$$
f(x)=f(0) m_{\sigma}(x), \quad g(x)=m_{\sigma}(x)
$$

for all $x \in S$. 
Proof. Putting $y=0$ in (35) we have

$$
|f(x)-f(0) g(x)| \leq \phi(0)
$$

for all $x \in S$. Choose a sequence $x_{n} \in S, n=1,2,3, \ldots$, such that $\left|g\left(x_{n}\right)\right| \rightarrow \infty$ as $n \rightarrow \infty$. Putting $x=x_{n}, n=$ $1,2,3, \ldots$, in (35), dividing the result by $\left|g\left(x_{n}\right)\right|$, letting $n \rightarrow$ $\infty$, and using (37) we have

$$
f(y)=\lim _{n \rightarrow \infty} \frac{f\left(x_{n}+\sigma y\right)}{g\left(x_{n}\right)}=\lim _{n \rightarrow \infty} \frac{f(0) g\left(x_{n}+\sigma y\right)}{g\left(x_{n}\right)}
$$

for all $y \in S$. Multiplying both sides of (38) by $f(x)$ and using (35) and (38) we have

$$
\begin{aligned}
f(y) f(x) & =\lim _{n \rightarrow \infty} \frac{f(0) g\left(x_{n}+\sigma y\right) f(x)}{g\left(x_{n}\right)} \\
& =\lim _{n \rightarrow \infty} \frac{f(0) f\left(x_{n}+\sigma y+\sigma x\right)}{g\left(x_{n}\right)}=f(0) f(x+y)
\end{aligned}
$$

for all $x, y \in S$. From (39) we have $f_{0}(x):=f(x) / f(0)$ is an exponential function, say $m$. Now, from (37) we can write

$$
f(x)=f(0) m(x), \quad g(x)=m(x)+r(x)
$$

for all $x \in S$, where $|r(x)| \leq \phi(0) /|f(0)|$ for all $x \in S$. Putting (40) in (35) and using the triangle inequality we have

$$
\begin{aligned}
|f(0) m(x)||m(\sigma y)-m(y)| & \leq \phi(y)+r(x)|f(y)| \\
& \leq \phi(y)+\frac{\phi(0)|f(y)|}{|f(0)|}
\end{aligned}
$$

for all $x, y \in S$. Since $m$ is unbounded, from (41) we have $m(\sigma y)=m(y)$ for all $y \in S$. Assume that $f$ satisfies (3) or $\phi$ satisfies (4). Choose a sequence $y_{n} \in S, n=1,2,3, \ldots$, such that $\left(1+\phi\left(y_{n}\right)\right) /\left|f\left(y_{n}\right)\right| \rightarrow 0$ as $n \rightarrow 0$. Putting $y=y_{n}, n=$ $1,2,3, \ldots$, in (35), dividing the result by $\left|f\left(y_{n}\right)\right|$, letting $n \rightarrow$ 0 , and using (37) we have

$$
g(x)=\lim _{n \rightarrow \infty} \frac{f\left(x+\sigma y_{n}\right)}{f\left(y_{n}\right)}=\lim _{n \rightarrow \infty} \frac{f(0) g\left(x+\sigma y_{n}\right)}{f\left(y_{n}\right)}
$$

for all $x \in S$. Multiplying both sides of (42) by $f(y)$ and using (35) and (42) we have

$$
\begin{aligned}
g(x) f(y) & =\lim _{n \rightarrow \infty} \frac{f(0) g\left(x+\sigma y_{n}\right) f(y)}{f\left(y_{n}\right)} \\
& =\lim _{n \rightarrow \infty} \frac{f(0) f\left(x+\sigma y+\sigma y_{n}\right)}{f\left(y_{n}\right)}=f(0) g(x+\sigma y)
\end{aligned}
$$

for all $x, y \in S$. Putting $x=0$ in (43), replacing $\sigma y$ by $x$, and dividing the result by $f(0)$ we have

$$
g(x)=g(0) f_{0}(\sigma x)=g(0) m_{\sigma}(\sigma x)=g(0) m_{\sigma}(x)
$$

for all $x \in S$. Putting $y=0$ in (35) and using (40) and (44) we get $g(0)=1$. This completes the proof.
Using Theorem 6 and applying the same method as in the proof of Corollary 3 we have the following.

Corollary 7. Let $P(x), x \in \mathbb{R}^{n}$, be a polynomial. Suppose that $f, g: \mathbb{R}^{n} \rightarrow \mathbb{C}$ are unbounded function satisfying

$$
|f(x+\sigma y)-g(x) f(y)| \leq|P(y)|
$$

for all $x, y \in \mathbb{R}^{n}$. Then there exists a $\sigma$-exponential function $m_{\sigma}: \mathbb{R}^{n} \rightarrow \mathbb{C}$ such that

$$
f(x)=f(0) m_{\sigma}(x), \quad g(x)=m_{\sigma}(x)
$$

for all $x \in \mathbb{R}^{n}$.

Using Theorem 6 and applying the same method as in the proof of Corollary 5 we have the following.

Corollary 8. Let $\gamma \in \mathbb{R}^{n}$ be fixed. Suppose that $f, g: \mathbb{R}^{n} \rightarrow \mathbb{C}$ are unbounded continuous function satisfying

$$
|f(x+\sigma y)-g(x) f(y)| \leq e^{\gamma \cdot y}
$$

for all $x, y \in \mathbb{R}^{n}$. Then there exists $c \in \mathbb{C}^{n}, \mathfrak{R}(c+c \sigma) \neq 0$ such that $f(x)=f(0) e^{(1 / 2)(c+c \sigma) \cdot x}$ for all $x \in \mathbb{R}^{n}$. If $(1 / 2) \Re(c+c \sigma) \neq$ $t \gamma$ for all $t \leq 1$, then one has

$$
f(x)=f(0) e^{(1 / 2)(c+c \sigma) \cdot x}, \quad g(x)=e^{(1 / 2)(c+c \sigma) \cdot x}
$$

for all $x \in \mathbb{R}^{n}$.

Theorem 9. Let $f, g: S \rightarrow \mathbb{C}$ be unbounded functions satisfying

$$
|f(x+\sigma y)-f(x) g(y)| \leq \phi(x)
$$

for all $x, y \in S$. Then there exists an unbounded exponential $m: S \rightarrow \mathbb{C}$ such that $f(x)=f(0) m(x)$ for all $x \in S$. In particular if $f$ satisfies (3) or $\phi$ satisfies (4), then one has

$$
f(x)=f(0) m(x), \quad g(x)=m(\sigma x)
$$

for all $x \in S$.

Proof. Putting $x=0$ in (49) we have

$$
|f(\sigma y)-f(0) g(y)| \leq \phi(0)
$$

for all $y \in S$. Choose a sequence $y_{n} \in S, n=1,2,3, \ldots$, such that $\left|g\left(y_{n}\right)\right| \rightarrow \infty$ as $n \rightarrow \infty$. Putting $y=y_{n}, n=1,2,3, \ldots$, in (49), dividing the result by $\left|g\left(y_{n}\right)\right|$, letting $n \rightarrow \infty$, and using (51) we have

$$
f(x)=\lim _{n \rightarrow \infty} \frac{f\left(x+\sigma y_{n}\right)}{g\left(y_{n}\right)}=\lim _{n \rightarrow \infty} \frac{f(0) g\left(\sigma x+y_{n}\right)}{g\left(y_{n}\right)}
$$

for all $x \in S$. Multiplying both sides of (52) by $f(y)$ and using (49) and (52) we have

$$
\begin{aligned}
f(y) f(x) & =\lim _{n \rightarrow \infty} \frac{f(0) f(y) g\left(\sigma x+y_{n}\right)}{g\left(y_{n}\right)} \\
& =\lim _{n \rightarrow \infty} \frac{f(0) f\left(y+x+\sigma y_{n}\right)}{g\left(y_{n}\right)}=f(0) f(x+y)
\end{aligned}
$$


for all $x, y \in S$. From (53) we have $f_{0}(x):=f(x) / f(0)$ is an exponential function, say $m$. Assume that $g$ satisfies (3) or $\phi$ satisfies (4). Choose a sequence $x_{n} \in S, n=1,2,3, \ldots$, such that $\left(1+\phi\left(x_{n}\right)\right) /\left|f\left(x_{n}\right)\right| \rightarrow 0$ as $n \rightarrow 0$. Putting $x=x_{n}, n=$ $1,2,3, \ldots$, in (49), dividing the result by $\left|f\left(x_{n}\right)\right|$, letting $n \rightarrow$ 0 , and using (51) we have

$$
g(y)=\lim _{n \rightarrow \infty} \frac{f\left(x_{n}+\sigma y\right)}{f\left(x_{n}\right)}=\lim _{n \rightarrow \infty} \frac{f(0) g\left(y+\sigma x_{n}\right)}{f\left(x_{n}\right)}
$$

for all $y \in S$. Multiplying both sides of (54) by $f(x)$ and using (49) and (54) we have

$$
\begin{aligned}
f(x) g(y) & =\lim _{n \rightarrow \infty} \frac{f(0) f(x) g\left(y+\sigma x_{n}\right)}{f\left(x_{n}\right)} \\
& =\lim _{n \rightarrow \infty} \frac{f(0) f\left(x+\sigma y+x_{n}\right)}{f\left(x_{n}\right)}=f(0) g(\sigma x+y)
\end{aligned}
$$

for all $x, y \in S$. Putting $y=0$ in (55), replacing $\sigma x$ by $x$, and dividing the result by $f(0)$ we have

$$
g(x)=g(0) f_{0}(\sigma x)=g(0) m(\sigma x)
$$

for all $x \in S$. Putting $x=0$ in (49) and using (56) we get $g(0)=1$. This completes the proof.

Using Theorem 9 we have the following.

Corollary 10. Let $P(x), x \in \mathbb{R}^{n}$, be a polynomial. Suppose that $f, g: \mathbb{R}^{n} \rightarrow \mathbb{C}$ are unbounded function satisfying

$$
|f(x+\sigma y)-f(x) g(y)| \leq|P(x)|
$$

for all $x, y \in \mathbb{R}^{n}$. Then there exists an exponential function $m: \mathbb{R}^{n} \rightarrow \mathbb{C}$ such that

$$
f(x)=f(0) m(x), \quad g(x)=m(\sigma x)
$$

for all $x \in \mathbb{R}^{n}$.

Corollary 11. Let $\gamma \in \mathbb{R}^{n}$ be fixed. Suppose that $f, g: \mathbb{R}^{n} \rightarrow$ $\mathbb{C}$ are unbounded continuous function satisfying

$$
|f(x+\sigma y)-f(x) g(y)| \leq e^{\gamma \cdot x}
$$

for all $x, y \in \mathbb{R}^{n}$. Then there exists $c \in \mathbb{C}^{n}, \mathfrak{R} c \neq 0$ such that $f(x)=f(0) e^{c \cdot x}$ for all $x \in \mathbb{R}^{n}$. If $\Re c \neq t \gamma$ for all $t \leq 1$, then we have

$$
f(x)=f(0) e^{c \cdot x}, \quad g(x)=e^{c \sigma \cdot x}
$$

for all $x \in \mathbb{R}^{n}$.

Theorem 12. Let $f, g: S \rightarrow \mathbb{C}$ be unbounded functions satisfying

$$
|f(x+\sigma y)-f(x) g(y)| \leq \phi(y)
$$

for all $x, y \in S$. Then $g$ is an exponential function. In particular, if $g$ satisfies the condition (3) or $\phi$ satisfies (4), then there exists an unbounded exponential $m: S \rightarrow \mathbb{C}$ such that

$$
f(x)=f(0) m(x), \quad g(x)=m(\sigma x)
$$

for all $x \in S$.
Proof. Choose a sequence $x_{n} \in S, n=1,2,3, \ldots$, such that $\left|f\left(x_{n}\right)\right| \rightarrow \infty$ as $n \rightarrow \infty$. Putting $x=x_{n}, n=1,2,3, \ldots$, in (61), dividing the result by $\left|f\left(x_{n}\right)\right|$, and letting $n \rightarrow \infty$ we have

$$
g(y)=\lim _{n \rightarrow \infty} \frac{f\left(x_{n}+\sigma y\right)}{f\left(x_{n}\right)}
$$

for all $y \in S$. Multiplying both sides of (63) by $g(x)$ and using (61) and (63) we have

$$
\begin{aligned}
g(y) g(x) & =\lim _{n \rightarrow \infty} \frac{f\left(x_{n}+\sigma y\right) g(x)}{f\left(x_{n}\right)} \\
& =\lim _{n \rightarrow \infty} \frac{f\left(x_{n}+\sigma y+\sigma x\right)}{f\left(x_{n}\right)}=g(x+y)
\end{aligned}
$$

for all $x, y \in S$. Therefore, $g$ is an exponential function, say $m$. Assume that $g$ satisfies (3) or $\phi$ satisfies (4). Choose a sequence $y_{n} \in S, n=1,2,3, \ldots$, such that $\left(1+\phi\left(y_{n}\right)\right) /$ $\left|g\left(y_{n}\right)\right| \rightarrow 0$ as $n \rightarrow \infty$. Putting $y=y_{n}, n=1,2,3, \ldots$, in (61), dividing the result by $\left|g\left(y_{n}\right)\right|$, and letting $n \rightarrow \infty$ we have

$$
f(x)=\lim _{n \rightarrow \infty} \frac{f\left(x+\sigma y_{n}\right)}{g\left(y_{n}\right)}
$$

for all $x \in S$. Multiplying both sides of (65) by $g(y)$ and using (61) and (65) we have

$$
\begin{aligned}
f(x) g(y) & =\lim _{n \rightarrow \infty} \frac{f\left(x+\sigma y_{n}\right) g(y)}{g\left(y_{n}\right)} \\
& =\lim _{n \rightarrow \infty} \frac{f\left(x+\sigma y_{n}+\sigma y\right)}{g\left(y_{n}\right)}=f(x+\sigma y)
\end{aligned}
$$

for all $x, y \in S$. Putting $x=0$ and replacing $y$ by $\sigma x$ in (66) we have $f(x)=f(0) m(\sigma x)$ for all $x \in S$. Replacing $m(x)$ by $m(\sigma x)$, we get (62). This completes the proof.

Using Theorem 12 we have the following.

Corollary 13. Let $P(x), x \in \mathbb{R}^{n}$, be a polynomial. Suppose that $f, g: \mathbb{R}^{n} \rightarrow \mathbb{C}$ are unbounded function satisfying

$$
|f(x+\sigma y)-f(x) g(y)| \leq|P(y)|
$$

for all $x, y \in \mathbb{R}^{n}$. Then there exists an exponential function $m: \mathbb{R}^{n} \rightarrow \mathbb{C}$ such that

$$
f(x)=f(0) m(x), \quad g(x)=m(\sigma x)
$$

for all $x \in \mathbb{R}^{n}$.

Corollary 14. Let $\gamma \in \mathbb{R}^{n}$ be fixed. Suppose that $f, g: \mathbb{R}^{n} \rightarrow$ $\mathbb{C}$ are unbounded continuous function satisfying

$$
|f(x+\sigma y)-f(x) g(y)| \leq e^{\gamma \cdot y}
$$

for all $x, y \in \mathbb{R}^{n}$. Then there exists $c \in \mathbb{C}^{n}, \mathfrak{R} c \neq 0$ such that $g(x)=e^{c \sigma \cdot x}$ for all $x \in S$. If $\Re c \neq t \gamma$ for all $t \leq 1$, then we have

$$
f(x)=f(0) e^{c \cdot x}, \quad g(x)=e^{c \sigma \cdot x}
$$

for all $x \in S$. 


\section{Applications}

In this section we consider the stability of (6) (8). A function $M:(0, \infty) \rightarrow \mathbb{R}$ is called multiplicative function provided that $M(x y)=M(x) M(y)$ for all $x, y>0$. Let $F(x+y i)=$ $f(x, y), G(x+i y)=g(x, y)$, and $\Phi(x+y i)=\phi(x, y)$ for all $(x, y) \in \mathbb{R}_{0}^{2}$. Then the functional inequalities (6) and (7) are converted to

$$
\begin{gathered}
|F(z \bar{w})-G(z) F(w)| \leq \Phi(z)[\text { resp., } \Phi(w)] \\
|F(z \bar{w})-F(z) G(w)| \leq \Phi(z)[\text { resp., } \Phi(w)]
\end{gathered}
$$

for all $z, w \in \mathbb{C}_{0}$.

Viewing $\mathbb{C}_{0}$ as a multiplicative group, letting $\sigma(z)=\bar{z}$, and applying Theorems 2 and 6 to the inequalities (71) we have the following.

Theorem 15. Let $f, g: \mathbb{R}_{0}^{2} \rightarrow \mathbb{R}$ be unbounded functions satisfying (6). Then $f, g$ are of the form

$$
\begin{gathered}
f(x, y)=f(1,0) M\left(\sqrt{x^{2}+y^{2}}\right), \\
g(x, y)=M\left(\sqrt{x^{2}+y^{2}}\right)
\end{gathered}
$$

for all $x, y \in \mathbb{R}$, where $M:(0, \infty) \rightarrow \mathbb{R}$ is a multiplicative function.

Applying Theorems 9 and 12 to the inequalities (72) we have the following.

Theorem 16. Let $f, g: \mathbb{R}_{0}^{2} \rightarrow \mathbb{R}$ be unbounded functions satisfying (7). Then $f, g$ are of the form

$$
\begin{gathered}
f(x, y)=f(1,0) M\left(\sqrt{x^{2}+y^{2}}\right) E\left(\tan ^{-1}\left(\frac{y}{x}\right)\right), \\
g(x, y)=M\left(\sqrt{x^{2}+y^{2}}\right) E\left(-\tan ^{-1}\left(\frac{y}{x}\right)\right)
\end{gathered}
$$

for all $(x, y) \in \mathbb{R}_{0}^{2}$, where $M:(0, \infty) \rightarrow \mathbb{R}$ is a multiplicative function and $E: \mathbb{R} \rightarrow \mathbb{R}$ is an exponential function satisfying $E(2 \pi)=1$.

Let $\mathbb{H}=\{a+b i+c j+d k \mid a, b, c, d \in \mathbb{R}\}$ be the set of quaternions. Recall that $i^{2}=j^{2}=k^{2}=-1, i j=k, j k=i$, $k i=j, j i=-k, k j=-i$, and $i k=-j$ and the conjugate of $q=a+b i+c j+d k \in \mathbb{U}$ is given by $q^{*}=a-b i-c j-d k$. We denote $\|q\|=\sqrt{q q^{*}}=\sqrt{a^{2}+b^{2}+c^{2}+d^{2}}$. Let $F(x+y i+$ $u j+v k)=f(x, y, u, v), G(x+i y+u j+v k)=g(x, y, u, v)$, and $\Phi(x+y i+u j+v k)=\phi(x, y, u, v)$ for all $(x, y, u, v) \in \mathbb{R}_{0}^{4}$. Then the functional inequalities (8) are converted to

$$
\left|F\left(q p^{*}\right)-G(q) F(p)\right| \leq \Phi(q)[\text { resp., } \Phi(p)]
$$

for all $p, q \in \mathbb{H} \backslash\{0\}$.

Applying Theorems 2 and 6 to the inequalities (75) we have the following.
Theorem 17. Let $f, g: \mathbb{R}_{0}^{4} \rightarrow \mathbb{R}$ be unbounded functions satisfying (8). Then $f, g$ are of the form

$$
\begin{gathered}
f(x, y, u, v)=f(1,0,0,0) M\left(\sqrt{x^{2}+y^{2}+u^{2}+v^{2}}\right), \\
g(x, y, u, v)=M\left(\sqrt{x^{2}+y^{2}+u^{2}+v^{2}}\right)
\end{gathered}
$$

for all $(x, y, u, v) \in \mathbb{R}_{0}^{4}$, where $M:(0, \infty) \rightarrow \mathbb{R}$ is a multiplicative function.

\section{Stability in $L^{\infty}$-Version}

Let $f: \mathbb{R}^{n} \rightarrow \mathbb{C}$ be a locally integrable function and $\sigma$ : $\mathbb{R}^{n} \rightarrow \mathbb{R}^{n}$ an involution. In this section, we consider an $L^{\infty}$ version of the stability of functional equation

$$
f(x+\sigma y)=f(x) f(y)
$$

for almost every $(x, y) \in \mathbb{R}^{2 n}$. More precisely, we study the functional inequality

$$
\|f(x+\sigma y)-f(x) f(y)\|_{L^{\infty}\left(\mathbb{R}^{2 n}\right)} \leq \epsilon .
$$

As is well known, inequality (78) implies

$$
\left|\int(f(x+\sigma y)-f(x) f(y)) \varphi(x, y) d x d y\right| \leq \epsilon\|\varphi\|_{L^{1}\left(\mathbb{R}^{2 n}\right)}
$$

for all $\varphi \in L^{1}\left(\mathbb{R}^{4}\right)$.

We first employ $\delta: \mathbb{R}^{n} \rightarrow \mathbb{R}$ defined by

$$
\delta(x)= \begin{cases}q e^{-\left(1-|x|^{2}\right)^{-1}}, & \text { if }|x|<1 \\ 0, & \text { if }|x| \geq 1\end{cases}
$$

where $q=\left(\int_{|x|<1} e^{-\left(1-|x|^{2}\right)^{-1}} d x\right)^{-1}$. It is easy to see that $\delta$ is an infinitely differentiable function with support $\{x:|x| \leq 1\}$.

Let $f$ be a locally integrable function on $\mathbb{R}^{n}$ and $\delta_{t}(x):=$ $t^{-n} \delta(x / t), t>0$. Then for each $t>0$,

$$
f * \delta_{t}(x)=\int_{\mathbb{R}^{n}} f(\xi) \delta_{t}(x-\xi) d \xi
$$

is a smooth function and $f * \delta_{t}(x) \rightarrow f(x)$ for almost every $x \in \mathbb{R}^{n}$ as $t \rightarrow 0^{+}$.

In the following, we exclude the case when $f(x)=0$ for almost every $x \in \mathbb{R}^{n}$.

Theorem 18. Let $f: \mathbb{R}^{n} \rightarrow \mathbb{C}$ satisfy (78). Then either there exists an unbounded exponential function $m: \mathbb{R}^{n} \rightarrow \mathbb{C}$ such that

$$
f(x)=m\left(\frac{x+\sigma x}{2}\right)
$$

for almost every $x \in \mathbb{R}^{n}$, or else

$$
\|f\|_{L^{\infty}\left(\mathbb{R}^{n}\right)} \leq \frac{1}{2}(1+\sqrt{1+4 \epsilon}) .
$$


If $\epsilon<1 / 4$, then either

$$
\frac{1}{2}(1+\sqrt{1-4 \epsilon}) \leq\|f\|_{L^{\infty}\left(\mathbb{R}^{n}\right)} \leq \frac{1}{2}(1+\sqrt{1+4 \epsilon})
$$

or

$$
\|f\|_{L^{\infty}\left(\mathbb{R}^{n}\right)} \leq \frac{1}{2}(1-\sqrt{1-4 \epsilon}) .
$$

Proof. Applying $\varphi\left(x^{\prime}, y^{\prime}\right)=\delta_{t}\left(x-x^{\prime}\right) \delta_{s}\left(y-y^{\prime}\right)$ in (79) we have

$$
\begin{aligned}
& \iint f\left(x^{\prime}+\sigma y^{\prime}\right) \delta_{t}\left(x-x^{\prime}\right) \delta_{s}\left(y-y^{\prime}\right) d x^{\prime} d y^{\prime} \\
& =\int f\left(z^{\prime}\right)\left(\int \delta_{t}\left(x-z^{\prime}+\sigma y^{\prime}\right) \delta_{s}\left(y-y^{\prime}\right) d y^{\prime}\right) d z^{\prime} \\
& =\int f\left(z^{\prime}\right)\left(\int \delta_{t}\left(x-z^{\prime}+\sigma y^{\prime}\right)\left(\delta_{s} \circ \sigma\right)\right. \\
& \left.\quad \times\left(\sigma y-\sigma y^{\prime}\right) d y^{\prime}\right) d z^{\prime} \\
& =\int f\left(z^{\prime}\right)\left(\int \delta_{t}\left(x-z^{\prime}+y^{\prime}\right)\left(\delta_{s} \circ \sigma\right)\left(\sigma y-y^{\prime}\right) d y^{\prime}\right) d z^{\prime} \\
& =\int f\left(z^{\prime}\right)\left(\int \delta_{t}\left(y^{\prime}\right)\left(\delta_{s} \circ \sigma\right)\left(x+\sigma y-z^{\prime}-y^{\prime}\right) d y^{\prime}\right) d z^{\prime} \\
& =\int f\left(z^{\prime}\right) \delta_{t} *(\delta \circ \sigma)_{s}\left(x+\sigma y-z^{\prime}\right) d z^{\prime} \\
& =\left(f * \delta_{t} *(\delta \circ \sigma)_{s}\right)(x+\sigma y) .
\end{aligned}
$$

We also have

$$
\begin{aligned}
& \iint f\left(x^{\prime}\right) f\left(y^{\prime}\right) \delta_{t}\left(x-x^{\prime}\right) \delta_{s}\left(y-y^{\prime}\right) d x^{\prime} d y^{\prime} \\
& \quad=\int f\left(y^{\prime}\right)\left(\int f\left(x^{\prime}\right) \delta_{t}\left(x-x^{\prime}\right) d x^{\prime}\right) \delta_{s}\left(y-y^{\prime}\right) d y^{\prime} \\
& =\left(f * \delta_{t}\right)(x) \int f\left(y^{\prime}\right)\left(f * \delta_{s}\right)\left(y-y^{\prime}\right) d y \\
& =\left(f * \delta_{t}\right)(x)\left(f * \delta_{s}\right)(y) .
\end{aligned}
$$

Thus, the inequality (78) is converted to the classical functional inequality

$$
\left|\left(f * \delta_{t} * \delta_{s}^{\sigma}\right)(x+\sigma y)-\left(f * \delta_{t}\right)(x)\left(f * \delta_{s}\right)(y)\right| \leq \epsilon
$$

for all $x, y \in \mathbb{R}^{n}$, where $\delta^{\sigma}=\delta \circ \sigma$.

Choosing $y_{0} \in \mathbb{R}, s_{0}>0$ such that $\left(f * \delta_{s_{0}}\right)\left(y_{0}\right) \neq 0$, putting $y=y_{0}, s=s_{0}$ in (88), using the triangle inequality, and dividing the result by $\left|\left(f * \delta_{s_{0}}\right)\left(y_{0}\right)\right|$ we have

$$
\left|\left(f * \delta_{t}\right)(x)\right| \leq \frac{\left|\left(f * \delta_{t} * \delta_{s_{0}}^{\sigma}\right)\left(x+\sigma y_{0}\right)\right|+\epsilon}{\left|\left(f * \delta_{s_{0}}\right)\left(y_{0}\right)\right|}
$$

for all $x \in \mathbb{R}^{n}$. Since $\left(f * \delta_{t} * \delta_{s_{0}}^{\sigma}\right)\left(x+\sigma y_{0}\right) \rightarrow\left(f * \delta_{s_{0}}^{\sigma}\right)\left(x+\sigma y_{0}\right)$ as $t \rightarrow 0^{+}$, it follows that

$$
F(x):=\limsup _{t \rightarrow 0^{+}}\left(f * \delta_{t}\right)(x)
$$

exists for all $x \in \mathbb{R}^{n}$. Since $\left(f * \delta_{t}\right)(x) \rightarrow f(x)$ for almost every $x \in \mathbb{R}^{n}$, it follows from (90) that

$$
F(x)=f(x)
$$

for almost every $x \in \mathbb{R}^{n}$.

Fixing $y \in \mathbb{R}^{n}$ and letting $s \rightarrow 0^{+}$so that $\left(f * \delta_{s}\right)(y) \rightarrow$ $F(y)$ in (88), we have

$$
\left|\left(f * \delta_{t}\right)(x+\sigma y)-\left(f * \delta_{t}\right)(x) F(y)\right| \leq \epsilon
$$

for all $x, y \in \mathbb{R}^{n}$. We first consider the case when $F$ is unbounded. Let $y_{n} \in \mathbb{R}^{n}, n=1,2,3, \ldots$, be a sequence such that $\left|F\left(y_{n}\right)\right| \rightarrow \infty$. Putting $y=y_{n}$ in (92), dividing the result by $\left|F\left(y_{n}\right)\right|$, and letting $n \rightarrow \infty$ we have

$$
\left(f * \delta_{t}\right)(x)=\lim _{n \rightarrow \infty} \frac{\left(f * \delta_{t}\right)\left(x+\sigma y_{n}\right)}{F\left(y_{n}\right)}
$$

for all $(x, y) \in \mathbb{R}^{n}$. Multiplying $F(y)$ in (93) and using (92) and (93) we have

$$
\begin{aligned}
\left(f * \delta_{t}\right)(x) F(y) & =\lim _{n \rightarrow \infty} \frac{\left(f * \delta_{t}\right)\left(x+\sigma y_{n}\right) F(y)}{F\left(y_{n}\right)} \\
& =\lim _{n \rightarrow \infty} \frac{\left(f * \delta_{t}\right)\left(x+\sigma y+\sigma y_{n}\right)}{F\left(y_{n}\right)} \\
& =\left(f * \delta_{t}\right)(x+\sigma y)
\end{aligned}
$$

for all $x, y \in \mathbb{R}^{n}, t>0$. Putting $x=0$ in (94) we have

$$
\left(f * \delta_{t}\right)(0) F(y)=\left(f * \delta_{t}\right)(\sigma y)
$$

for all $y \in \mathbb{R}^{n}, t>0$. From (95) we have $\left(f * \delta_{t}\right)(0) \neq 0$ for some $t>0$. Putting (95) in (94) we have

$$
F(\sigma x) F(y)=F(y+\sigma x)
$$

for all $x, y \in \mathbb{R}^{n}$. From (96) $F$ is an exponential function. Now, we prove that

$$
F(x)=F(\sigma x)
$$

for all $x \in \mathbb{R}^{n}$. In view of (94), replacing $\left(f * \delta_{t}\right)(x)$ by $(f *$ $\left.\delta_{t}\right)(0) F(\sigma x)$ and $\left(f * \delta_{s}\right)(y)$ by $\left(f * \delta_{s}\right)(0) F(\sigma y)$ in (88) and letting $s \rightarrow 0^{+}$so that $\left(f * \delta_{s}\right)(0) \rightarrow F(0)$ we have

$$
\left|\left(f * \delta_{t}\right)(x+\sigma y)-\left(f * \delta_{t}\right)(0) F(\sigma x) F(\sigma y)\right| \leq \epsilon
$$

for all $x, y \in \mathbb{R}^{n}, t>0$. Using (95) and (98) we have

$$
\left|\left(f * \delta_{t}\right)(0) F(y+\sigma x)-\left(f * \delta_{t}\right)(0) F(\sigma x) F(\sigma y)\right| \leq \epsilon .
$$


Letting $t \rightarrow 0^{+}$in $(99)$ so that $\left(f * \delta_{t}\right)(0) \rightarrow F(0)$ we have

$$
|F(y+\sigma x)-F(\sigma x) F(\sigma y)| \leq \epsilon
$$

for all $x, y \in \mathbb{R}$. Since $F$ is an exponential function, it follows from (100) that

$$
|F(\sigma x)||F(y)-F(\sigma y)| \leq \epsilon
$$

for all $x, y \in \mathbb{R}^{n}$. Since $F$ is unbounded, from (101) we have $F(y)=F(\sigma y)$ for all $y \in \mathbb{R}^{n}$. Now, $F$ is written in the form

$$
\begin{aligned}
F(x) & =F\left(\frac{x}{2}+\frac{x}{2}\right)=F\left(\frac{x}{2}\right) F\left(\frac{x}{2}\right) \\
& =F\left(\frac{x}{2}\right) F\left(\frac{\sigma x}{2}\right)=F\left(\frac{x+\sigma x}{2}\right)
\end{aligned}
$$

for all $x, y \in \mathbb{R}^{n}$. Conversely, let $F(x)=m((x+\sigma x) / 2)$, where $m: \mathbb{R}^{n} \rightarrow \mathbb{C}$ is an arbitrary exponential function. Then $F$ is an exponential function satisfying $F(x)=F(\sigma x)$ for all $x \in \mathbb{R}^{n}$. Thus, we get (82). From now on, we assume that $F$ is bounded, say $|F(x)| \leq M$ for all $x \in \mathbb{R}^{n}$. Then, it follows from (91) that $\|f\|_{L^{\infty}\left(\mathbb{R}^{n}\right)} \leq M$. Thus, we have

$$
\begin{aligned}
\left|\left(f * \delta_{t}\right)(x)\right| & =\left|\int f\left(x^{\prime}\right) \delta\left(x-x^{\prime}\right) d x^{\prime}\right| \\
& \leq M \int\left|\delta\left(x-x^{\prime}\right)\right| d x^{\prime}=M
\end{aligned}
$$

for all $x \in \mathbb{R}^{n}$. From the inequality (92), using the method in Theorem 10 of [1] we have

$$
\left|\left(f * \delta_{t}\right)(x)(|F(y)|-1)\right| \leq \epsilon
$$

for all $x, y \in \mathbb{R}^{n}, t>0$. Fixing $x \in \mathbb{R}^{n}$ and letting $t \rightarrow 0^{+}$in (104) we have

$$
|F(x)(|F(y)|-1)| \leq \epsilon
$$

for all $x, y \in \mathbb{R}$. From (105), using the method in Theorem 10 of [1] we have

$$
|F(x)| \leq \frac{1}{2}(1+\sqrt{1-4 \epsilon})
$$

for all $x \in \mathbb{R}$, and if $\epsilon<1 / 4$, then we have either

$$
\frac{1}{2}(1-\sqrt{1+4 \epsilon}) \leq|F(x)| \leq \frac{1}{2}(1+\sqrt{1+4 \epsilon})
$$

for all $x \in \mathbb{R}$ or

$$
|F(x)| \leq \frac{1}{2}(1-\sqrt{1-4 \epsilon})
$$

for all $x \in \mathbb{R}$. Since $f(x)=F(x)$ almost every $x \in \mathbb{R}^{n}$, we get (83), (84), and (85). This completes the proof.

\section{Conflict of Interests}

The author declares that there is no conflict of interests regarding the publication of this paper.

\section{Acknowledgments}

This work was supported by Basic Science Research Program through the National Foundation of Korea (NRF) funded by the Korea Government (no. 2012R1A1A008507). The author expresses his deep thanks to the referee for useful comments on some possible generalization of Theorem 2 .

\section{References}

[1] J. Chung and S.-Y. Chung, "Stability of exponential functional equations with involutions," Journal of Function Spaces, vol. 2014, Article ID 619710, 9 pages, 2014.

[2] J. Chung and J. Chang, "On two functional equations originating from number theory," to appear in Proceedings of the Indian Academy of Science.

[3] K. B. Houston and P. K. Sahoo, "On two functional equations and their solutions," Applied Mathematics Letters, vol. 21, no. 9, pp. 974-977, 2008.

[4] S. Jung and J. Bae, "Some functional equations originating from number theory," Proceedings of the Indian Academy of SciencesMathematical Sciences, vol. 113, no. 2, pp. 91-98, 2003.

[5] G. H. Kim and P. K. Sahoo, "Stability of some functional equations related to distance measures-I," Applied Mathematics Letters, vol. 24, no. 6, pp. 843-849, 2011.

[6] J. A. Baker, "The stability of cosine functional equation," Proceedings of the American Mathematical Society, vol. 80, no. 3, pp. 411-416, 1980.

[7] A. Bahyrycz, "On solutions of the second generalization of d'Alembert's functional equation on a restricted domain," Applied Mathematics and Computation, vol. 223, pp. 209-215, 2013.

[8] A. Bahyrycz and J. Brzdęk, "On solutions of the d'Alembert equation on a restricted domain," Aequationes Mathematicae, vol. 85, no. 1-2, pp. 169-183, 2013.

[9] A. Bahyrycz and J. Brzdek, "A note on d'Alembert's functional equation on a restricted domain," Aequationes Mathematicae, 2014.

[10] J. Brzdęk, "On solutions of a generalization of the Reynolds functional equation," Demonstratio Mathematica, vol. 41, no. 4, pp. 859-868, 2008.

[11] J. Brzdek, A. Najdecki, and B. Xu, "Two general theorems on superstability of functional equations," Aequationes mathematicae, 2014.

[12] J. Chudziak and J. Tabor, "On the stability of the Gołab-Schinzel functional equation," Journal of Mathematical Analysis and Applications, vol. 302, no. 1, pp. 196-200, 2005.

[13] J. Chung, "On an exponential functional inequality and its distributional version," Canadian Mathematical Bulletin, 2014.

[14] J. G. Dhombres and R. Ger, "Conditional Cauchy equations," Glasnik Matematicki, vol. 13, no. 33, pp. 39-62, 1978.

[15] R. Ger and P. Šemrl, "The stability of the exponential equation," Proceedings of the American Mathematical Society, vol. 124, no. 3, pp. 779-787, 1996.

[16] A. Najdecki, "On stability of a functional equation connected with the Reynolds operator," Journal of Inequalities and Applications, vol. 2007, Article ID 79816, 3 pages, 2007. 
[17] N. Brillouët-Belluot, J. Brzdęk, and K. Ciepliński, "On some recent developments in Ulam's type stability," Abstract and Applied Analysis, vol. 2012, Article ID 716936, 41 pages, 2012.

[18] J. Brzdęk and K. Ciepliński, "Hyperstability and superstability," Abstract and Applied Analysis, vol. 2013, Article ID 401756, 13 pages, 2013.

[19] D. H. Hyers, G. Isac, and T. M. Rassias, Stability of Functional Equations in Several Variables, Progress in Nonlinear Differential Equations and Their Applications, Birkhäuser, Basel, Switzerland, 1998. 


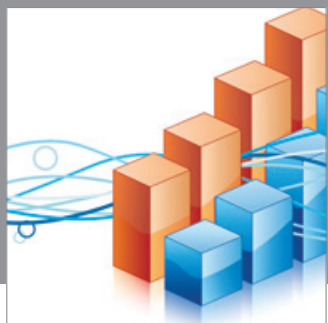

Advances in

Operations Research

mansans

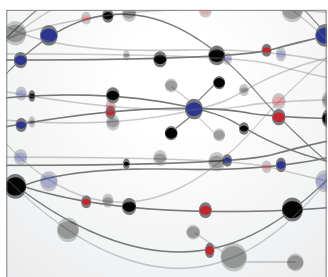

The Scientific World Journal
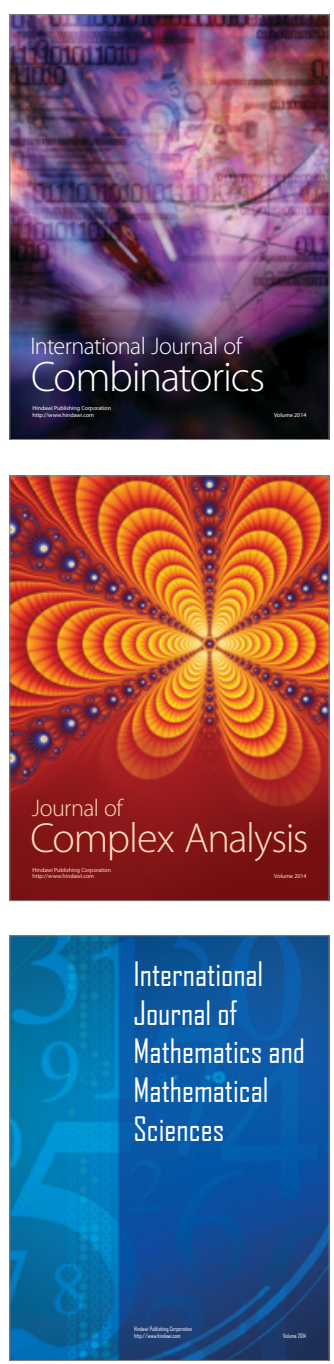
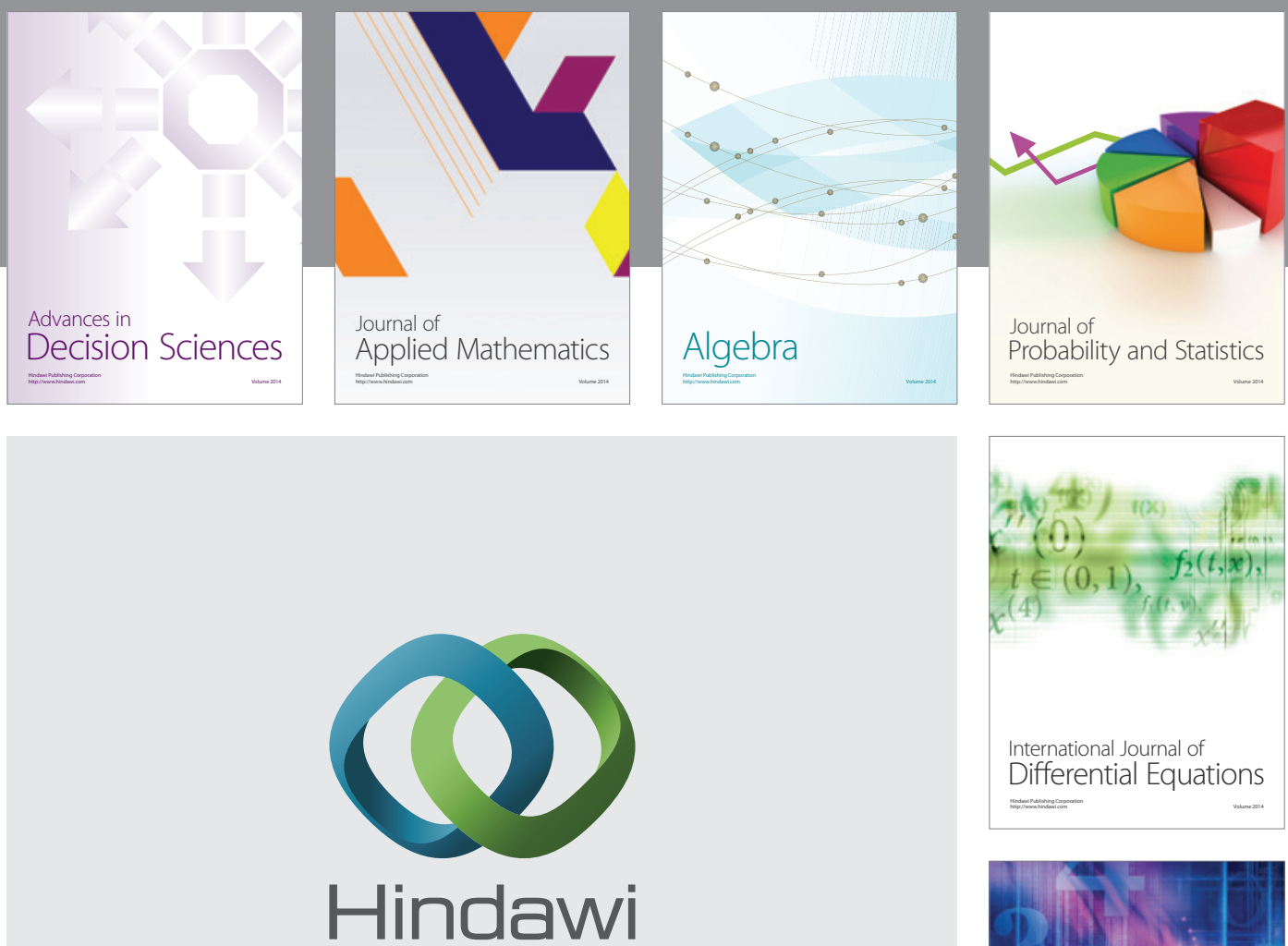

Submit your manuscripts at http://www.hindawi.com
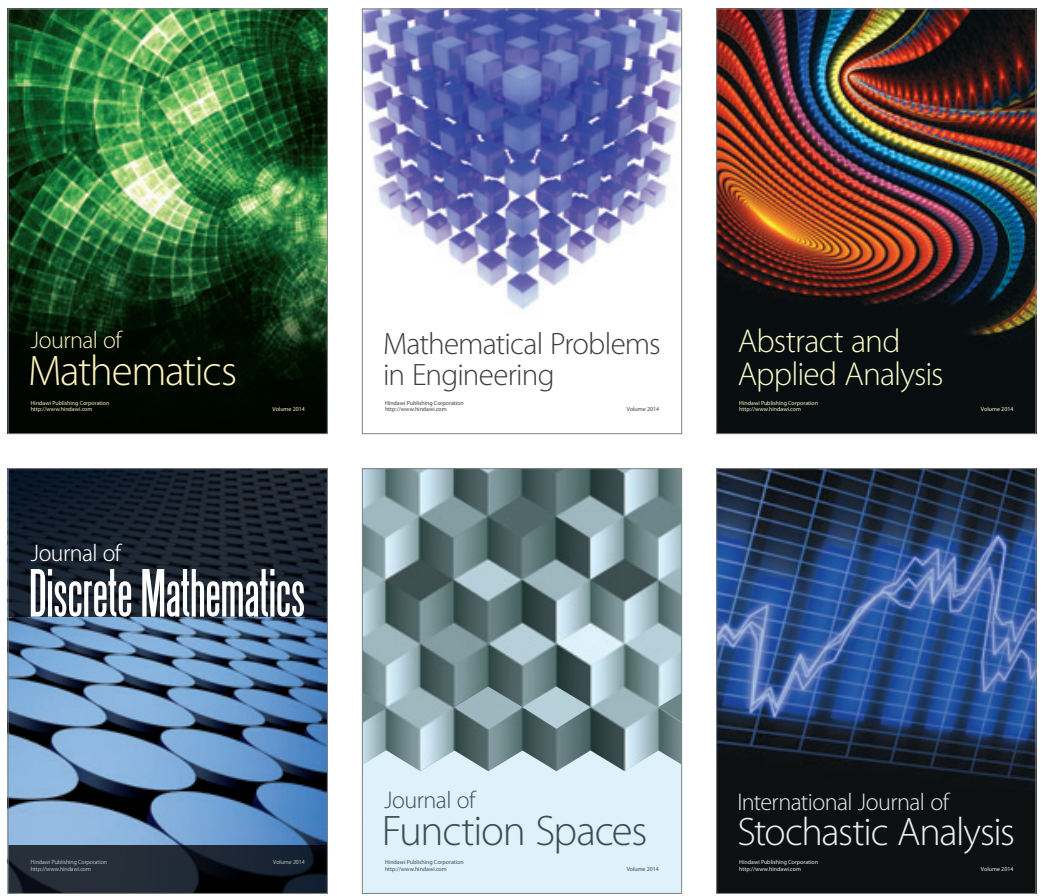

Journal of

Function Spaces

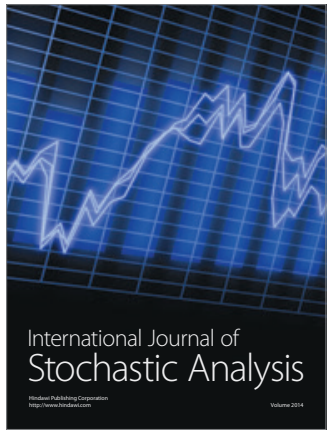

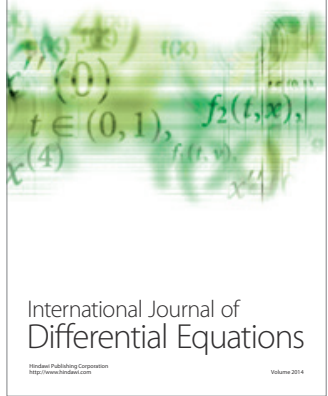
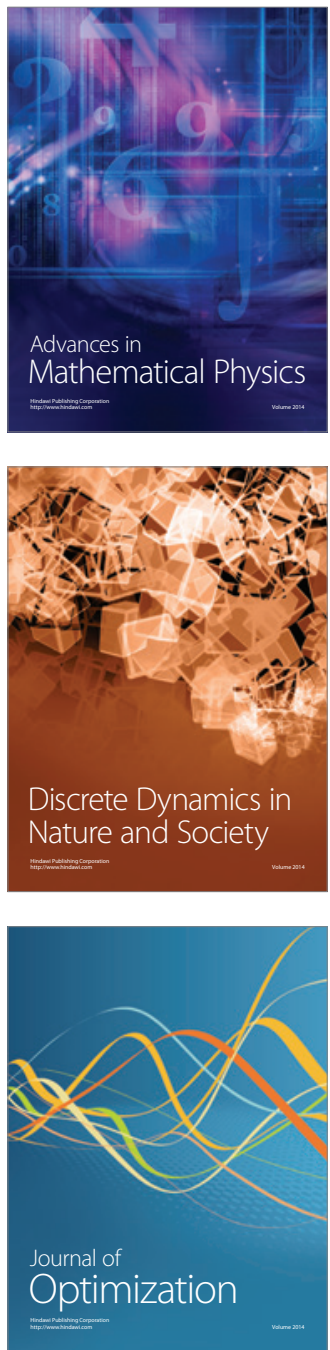